\title{
LIVER
}

\section{Hepatic haemangiomas: possible association with female sex hormones}

\author{
V Glinkova, O Shevah, M Boaz, A Levine, H Shirin
}

Gut 2004;53:1352-1355. doi: 10.1136/gut.2003.038646

See end of article for authors' affiliations .....................

Correspondence to: Dr H Shirin, Department of Gastroenterology, The E Wolfson Medical Center, Holon 58100, Israel; shirin@ wolfson.health.gov.il

Revised version received 15 February 2004 Accepted for publication 19 February 2004

\begin{abstract}
Background and aims: The association of hepatic haemangiomas with female sex hormones is not entirely clear. We prospectively evaluated the impact of female sex hormones on the natural history of liver haemangiomas.

Methods: We followed 94 women with 181 haemangiomas diagnosed by ultrasound for a period of 1-17 years (mean 7.3 (5.5) years). The location, number, size, and ultrasonographic pattern of the lesions were evaluated. Patients were also evaluated by questionnaire for gynaecological and reproductive history. We compared the change in number and size of haemangiomas in patients who received or did not receive exogenous hormonal treatment.

Results: Age at first period was inversely associated with the size of haemangiomas $(r=0.181, p=0.015)$ while age at menopause was positively correlated with the number of haemangiomas detected at first ultrasound $(r=0.542, p<0.0001)$. During follow up, no change in the ultrasonographic pattern or number of haemangiomas was observed. An increase in the size of the lesions was demonstrated in 5/22 (22.7\%) hormone therapy exposed patients compared with $7 / 72(9.7 \%)$ controls. Three variables (ultrasonographic pattern, number of haemangiomas, and hormone therapy) predicted whether or not a given haemangioma would increase in size. A hypoechoic pattern increased the risk of progression while a hyperechoic pattern decreases that risk $(p=0.003)$. The number of haemangiomas was inversely associated with the likelihood of progression $(p=0.006)$ and hormone therapy increased the risk of haemangioma enlargement $(p=0.05)$.

Conclusions: Hepatic haemangiomas seem to be influenced by both endogenous and exogenous female sex hormones although significant enlargement occurs only in a minority of patients. Consequently, routine liver ultrasound follow up in women with hepatic haemangiomas receiving hormone therapy appears appropriate.
\end{abstract}

$\mathrm{H}$ epatic haemangiomas are benign hepatocellular neoplasms. They are most commonly found in women, with a female:male ratio of up to $5: 1$, emphasising the importance of excess of female sex hormones in these tumours. ${ }^{1-3}$ Haemangiomas of the liver are usually detected incidentally by ultrasound. Lesions smaller than $6 \mathrm{~cm}$ in diameter characteristically demonstrate a well demarcated, homogeneous, hyperechoic ultrasonographic pattern that may establish the diagnosis in $80 \%$ of patients. ${ }^{4}$ Larger haemangiomas are more heterogenous, often complicated by thrombosis, calcification, or haemorrhage, and require further imaging by computed tomography (CT) scan or magnetic resonance imaging (MRI).

Ultrasound surveillance may detect a spontaneous increase in the size of hepatic haemangiomas. ${ }^{35}$ The natural history over long term follow up has not been previously reported. Several studies have reported that haemangioma size increases during pregnancy and during oestrogen therapy, suggesting a causative role for oestrogen in tumour growth ${ }^{6-8}$; however, this association has not consistently been found. ${ }^{3}$ Hepatic adenomas have been reported to express both oestrogen and progesterone receptors in approximately $73 \%$ of patients ${ }^{9}$ although two case reports demonstrating sex hormone associated growth of hepatic haemangiomas failed to demonstrate oestrogen receptors in these tumours. ${ }^{6}{ }^{10}$

The aim of the present study was to evaluate the natural history of liver haemangiomas among women, identify prognostic variables linked to growth over time, and to determine if haemangioma growth or number is affected by endogenous or exogenous female sex hormones.

\section{PATIENTS AND METHODS}

Haemangiomas were detected incidentally from ultrasound check ups for other diseases, mainly gall bladder disease and nephrolithiasis. Between 1986 and 2003, 94 women were diagnosed with liver haemangioma and were followed for 1-17 years (mean 7.3 (5.5) years) in the Hepatogastroenterology Clinic at the "St Marina" University Hospital, Varna, Bulgaria. All patients underwent imaging follow up every three months in the first year and yearly thereafter. Location, number, size, and ultrasonographic pattern of the lesions were evaluated. Patients were diagnosed by ultrasound with a real time sector, curved array, and linear array scanners with 3.0, 3.5, 3.7, 5.0, and 6.0 $\mathrm{MHz}$ probes. Criteria for classifying a focal hepatic lesion as a haemangioma included a characteristic hyperechoic well delineated ultrasound pattern, absence of clinical symptoms, and normal liver enzymes. A single reader attempted to determine the number, size, location, and ultrasonographic features of all haemangiomas. Twenty six of these patients with ultrasonographically unclear findings, cases clinically doubtful for malignancy, or women with a history of other malignancies underwent CT with bolus intravenous contrast medium enhancement. Ten patients who had doubtful CT for malignancy underwent fine needle aspiration (FNA) biopsy. All patients with CT or FNA findings incompatible with haemangioma were excluded. None of the patients underwent MRI or tagged red blood cell studies.

Abbreviations: $\mathrm{CT}$, computed tomography; MRI, magnetic resonance imaging; FNA, fine needle aspiration; OR, odds ratio 
In addition to radiological evaluation, patients were evaluated by questionnaire that included questions regarding: (a) age at first menstrual period, (b) number of pregnancies and deliveries, (c) age at menopause, and (d) hormone therapy (contraceptives, hormone replacement therapy, and other hormonal therapies). Subjects were characterised on the basis of exposure to exogenous oestrogen treatment, such that exposed subjects comprised the hormone therapy group and unexposed subjects served as the control group. Changes in the number and size of the haemangiomas were compared by exogenous oestrogen treatment exposure. Enlargement was defined as an increase of at least $0.5 \mathrm{~cm}$ during the observation period. Significant enlargement was defined as an increase in size $>1 \mathrm{~cm}$ during follow up with a final diameter $>2 \mathrm{~cm}$. Large haemangiomas were defined as haemangiomas with a diameter $>4 \mathrm{~cm}$.

\section{Data analysis}

Analysis of the data was carried out using SPSS statistical analysis software (SPSS Inc., Chicago, Illinois, USA, 1999). For continuous variables such as age, haemangioma size, and duration of follow up, descriptive statistics were calculated and are reported as mean (SD). Categorical variables such as exposure to hormone therapy and location or pattern of ultrasound were described using frequency distributions. Distributions of continuous variables were tested for normality using the Komolgorov-Smirnov test. Variables with distributions differing significantly from normal, such as number of pregnancies and age at first menstrual period, were compared by treatment assignment using the MannWhitney non-parametric $\mathrm{U}$ test. The $t$ test for independent samples was used to detect differences in means of normally distributed continuous variables using exposure to hormone replacement therapy as the categorical variable. The $\chi^{2}$ test (exact, when possible) was used to detect differences in categorical variables by exposure to hormone replacement therapy. Associations between variables were described using Spearman's rho. Progression of haemangioma at the end point was described using logistic regression analysis, and odds ratios (OR) for progression with 95\% confidence intervals (CI) were calculated. All tests were considered significant at $\mathrm{p}<0.05$.

\section{RESULTS}

\section{Patients}

Mean age at the first examination was 48.4 (9.3) years (range 29-72). Characteristics of the women with liver haemangioma in the two groups, with and without hormone therapy, are summarised in table 1. Mean age at first menstrual period was 13.5 (1.39) years (range 11-16). Subjects had a median of 3.0 (range 0-18) pregnancies and 2.0 (range 0-5) deliveries. No women became pregnant during the study. At baseline, 58/94 (61.7\%) women reported

Table 1 Characteristics of the women with liver haemangiomas

\begin{tabular}{llll}
\hline & $\begin{array}{l}\text { Control } \\
\text { group }\end{array}$ & $\begin{array}{l}\text { Hormone } \\
\text { therapy } \\
\text { group }\end{array}$ & All \\
\hline No of patients & 72 & 22 & 94 \\
Age (y) & $49.9(9.0)$ & $44.4(9.1)$ & $48.4(9.3)$ \\
Age at 1st period (y) & $13.5(1.39)$ & $13.3(1.1)$ & $13.5(1.3)$ \\
Pregnancies (median) & $3(1,11)$ & $2(0,18)$ & $3(0,18)$ \\
Deliveries (median) & $2(0,4)$ & $2(0,5)$ & $2(0,5)$ \\
Women in menopause (n) & 49 & 9 & 58 \\
Age at menopause (y) & $48.8(3.0)$ & $50.2(1.4)$ & $49.0(2.9)$ \\
Follow up (y) & $7.5(5.7)$ & $6.9(5.1)$ & $7.3(5.5)$ \\
\hline
\end{tabular}
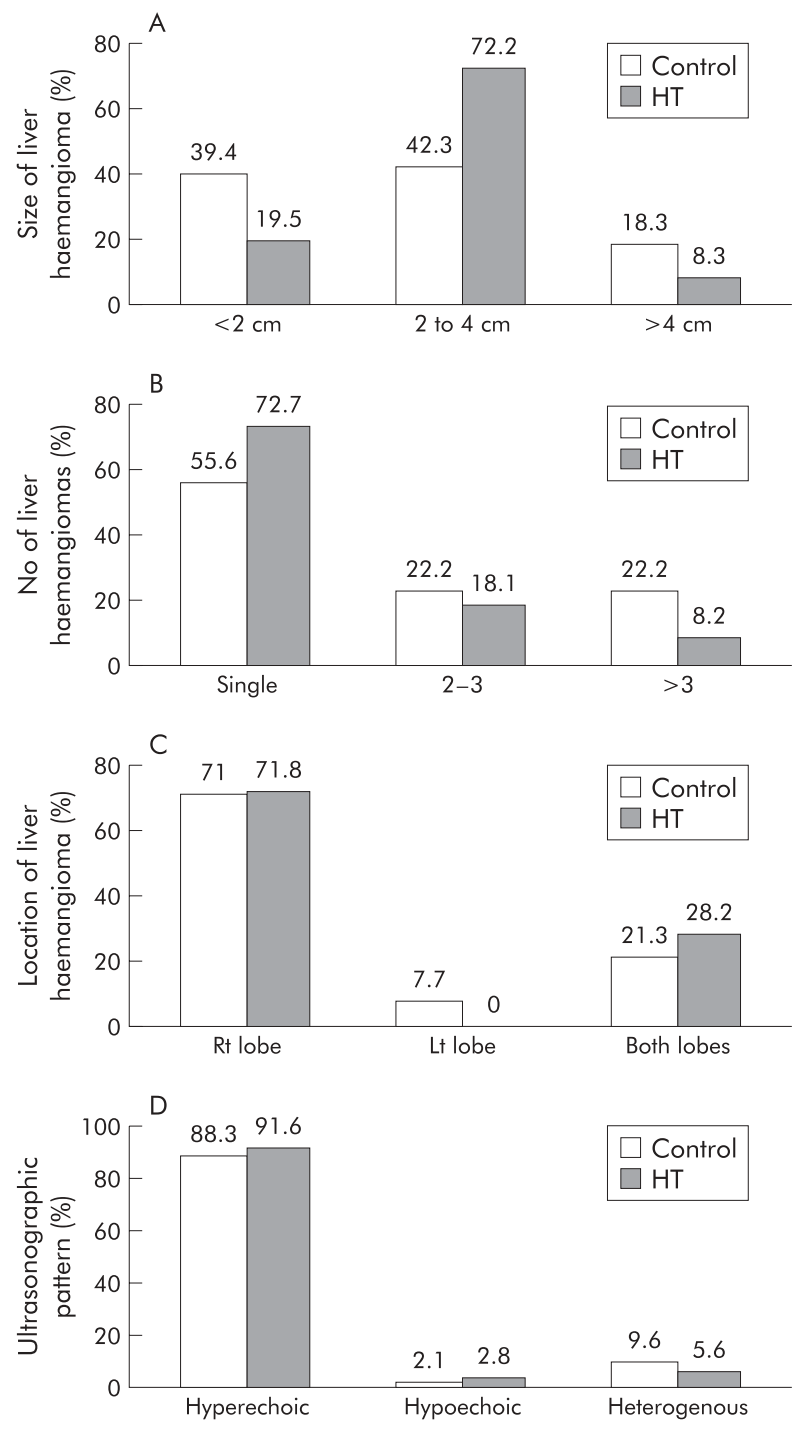

Figure 1 Comparison of the size (A), number (B), location (C), and ultrasonographic pattern (D) of the lesions between the control group and the hormone therapy $(\mathrm{HT})$ group.

having gone through the menopause (some of them started the menopause during follow up). There were 72 women in the control group and 22 women who had received some exogenous female sex hormone therapy. All women had initiated hormonal treatment before the study, with a mean duration of 35 months (range 3-90). Hormone therapy included contraceptives $(11 / 22 ; 50 \%)$, postmenopausal hormone replacement therapy $(4 / 22 ; 18.1 \%)$, and progesterone preparations for regulation of menstrual periods (7/22; $31.9 \%)$.

\section{Characteristics of liver haemangiomas}

A total of 181 haemangiomas were detected. As shown in fig $1,128 / 181(70.8 \%)$ were located in the right lobe of the liver, $11 / 181(6.0 \%)$ in the left lobe, and $42 / 181(23.2 \%)$ in both lobes. Also shown in figure 1 is lesion size distribution. As can be seen, most lesions were smaller than $4 \mathrm{~cm}$. Distribution by size showed that $92 / 181(50.8 \%)$ haemangiomas were $>2 \mathrm{~cm}$ in size. Most subjects (56/94) had a single lesion, 20/94 (21.2\%) 2-3 lesions, and 18/94 (19.2\%) multiple (>3) lesions. Ultrasonographically, most haemangiomas (161/181; 88.9\%) demonstrated a hyperechoic pattern, 


\begin{tabular}{|c|c|c|c|c|c|c|}
\hline Patient No & $\begin{array}{l}\text { Size at 1st } \\
\text { US }(\mathrm{cm})\end{array}$ & $\begin{array}{l}\text { Size at last } \\
\text { US }(\mathrm{cm})\end{array}$ & $\Delta(\mathrm{cm})$ & US pattern & Hormone therapy & Follow up (y) \\
\hline 1 & 1.5 & 2.4 & 0.7 & Hyper & - & 3 \\
\hline 2 & 0.5 & 1.6 & 1.1 & Hyper & - & 2 \\
\hline 3 & 1.5 & 2.0 & 0.5 & Hyper & - & 5 \\
\hline 4 & 1.7 & 2.8 & 1.1 & Нуро & - & 12 \\
\hline 5 & 3.5 & 4.0 & 0.5 & Нуро & - & 17 \\
\hline 6 & 10 & 16 & 6.0 & Hetero & - & 16 \\
\hline 7 & 2 & 6 & 4.0 & Hyper & - & 4 \\
\hline 8 & 10 & 13 & 3.0 & Hetero & HRT & 17 \\
\hline 9 & 1.6 & 2.4 & 0.8 & Hyper & Contraceptives & 10 \\
\hline 10 & 2 & 4 & 2.0 & Hyper & Progesterone & 5 \\
\hline 11 & 0.5 & 1.2 & 0.7 & Hyper & Progesterone & 7 \\
\hline 12 & 4 & 8 & 4.0 & Hyper & Contraceptives & 7 \\
\hline
\end{tabular}

$16 / 181(8.8 \%)$ a heterogenous pattern, and only $4 / 181(2.3 \%)$ were hypoechoic (fig 1). During follow up, no change in the ultrasonographic pattern of the lesions was demonstrated. However, the size of the haemangiomas differed significantly by echoic appearance, such that haemangiomas with a heterogenous pattern were larger than those with a hyperechoic pattern (13 (3) cm $v 3.2$ (2) cm, respectively; $\mathrm{p}<0.0001$ ).

Age at first menstrual period was inversely associated with size of haemangioma at first ultrasound $(r=0.181$, $\mathrm{p}=0.015)$. Age at menopause was positively correlated with number of haemangiomas detected at first ultrasound $(r=0.542, \mathrm{p}<0.0001)$. However, age at menopause was also marginally inversely associated with size of haemangioma at first ultrasound $(r=0.177, \mathrm{p}=0.054)$.

\section{Change in number/size of haemangiomas}

No change in the number of haemangiomas was observed during the study. Change in the size was determined in 12/94 patients $(12.7 \%)$, of whom five had received hormone therapy (table 2). Three variables (ultrasonographic pattern, number of haemangiomas, and hormone therapy) predicted whether or not a given haemangioma would increase in size although for hormone therapy this was only marginally significant. Specifically, hypoechoic pattern increased the risk of progression (OR 3.7 (95\% CI 1.5-9.0); p=0.0035). Haemangioma number was inversely associated with the likelihood that progression would occur (OR 0.27 (95\% CI $0.11-0.68) ; p=0.006)$. Finally, hormone therapy increased the risk of haemangioma progress (OR 3.02 (95\% CI 0.999.12); $\mathrm{p}=0.05$ ). A multivariate model using ultrasonographic pattern, number of haemangioma, and forcing age into the model revealed similar accuracy, with overall significance of $\mathrm{p}<0.0001$. Significant enlargement, as previously defined, occurred in only 6/181 haemangiomas, five of these in haemangiomas $>2 \mathrm{~cm}$ at the initial observation. The solitary patient with a haemangioma $<2 \mathrm{~cm}$ and $>1 \mathrm{~cm}$ had only $1.1 \mathrm{~cm}$ of growth over 12 years of follow up. Nevertheless, the increase in the size of the haemangiomas did not correlate with the initial size of the haemangiomas.

\section{DISCUSSION}

In this study, we attempted to evaluate the natural history of liver haemangiomas, influence of female sex hormones on growth characteristics, and diagnostic variables that can predict growth behaviour. During a long term follow up, an increase in size was observed in only $12.7 \%$ of women although this rate was higher than that found in previous reports. ${ }^{31}$ Significant growth over time was even rarer, occurring in only $6.3 \%$ of patients. Parameters independently linked to growth included the ultrasonographic pattern (hypoechoic), presence of single or few haemangiomas on ultrasound, and hormone therapy.

Our results indicate that there is an association between female hormonal factors, such as age at first menstrual period and age at menopause, and number of hepatic haemangiomas. An increase in haemangioma size occurred in $22.7 \%$ of hormone therapy exposed patients compared with $9.7 \%$ of controls, representing more than a twofold increase, although the numbers were small. This effect was also evident when haemangiomas were analysed as a cohort. Specifically, $13.8 \%$ of haemangiomas identified in hormone treatment exposed women progressed compared with $4.8 \%$ of haemangiomas in controls. It is important to note that in almost $60 \%(7 / 12)$ of female patients with growth of haemangiomas there was no history of exposure to exogenous hormonal therapy.

In previous studies, the prevalence of hepatic haemangiomas varied between $1.4 \%$ and $20 \%,{ }^{12-14}$ with multiple lesions occurring in $9-22 \%$ of patients. ${ }^{13}{ }^{15}$ Even though very little is known about the aetiology of liver haemangioma, it has been suggested that haemangiomas are not neoplasms but congenital hamartomas. ${ }^{13}$ Consequently, these lesions grow due to dilatation or ectasia of the vascular channels rather than proliferation of endothelial cells. ${ }^{10}$ In this regard, other possible mechanisms for enlargement of cavernous haemangioma, such as bleeding or intratumoral thrombosis, have been proposed. ${ }^{16}{ }^{17}$ Alternatively, others have suggested that haemangiomas are true neoplasms and therefore their enlargement is accompanied by proliferation of new blood vessels in the lesion. ${ }^{18}$

The high prevalence of these lesions in women, together with several publications on rapid growth of haemangiomas during pregnancy and after exogenous oestrogen or steroid administration, have suggested an important role for female sex hormones in the progression of these lesions. ${ }^{1-3}$ Conter and Longmire documented four recurrences of giant haemangiomas, three of which occurred in women who had been receiving oral oestrogen therapy prior to and after their original surgery. ${ }^{7}$ Takahashi et al described enlargement of multiple haemangiomas during seven years of steroid therapy for myasthenia gravis. ${ }^{19}$ Furthermore, in patients with liver focal nodular hyperplasia, the development of which is suspected to be promoted by oral contraceptive use, ${ }^{20}$ the prevalence of hepatic haemangiomas is elevated..$^{21} 22$

Based on clinical and experimental cardiovascular surgery data, it has been speculated that steroids may affect the micro architecture of the vascular wall, which is part of the cavernous haemangioma. ${ }^{23}$ This may be mediated at least partially by the inhibitory effect of prednisone on collagen biosynthesis. ${ }^{24}$ On the other hand, data supporting the fact that steroids may initiate or promote angiogenesis or 
neovascularisation is very limited. Schnaper et al reported that oestrogen enhances endothelial cell proliferation, migration, and organisation into capillary-like structures in vitro and augments experimental angiogenesis in vivo. ${ }^{25}$ In contrast, in vitro studies suggest that certain steroids may inhibit angiogenesis. ${ }^{26}$ These controversial reports demonstrate that the mechanism by which oestrogens may regulate endothelial cells turnover in liver tumours still needs to be clarified.

In summary, endogenous and exogenous female sex hormones seem to play a role in the pathogenesis of liver haemangiomas although significant enlargement occurs in only a minority of patients. None of our patients developed symptoms or complications related to the presence of these haemangiomas, questioning the need for observation, especially in patients with no risk for significant enlargement over time. However, routine liver ultrasound follow up in women with hepatic haemangiomas receiving hormone therapy appears appropriate.

\section{Authors' affiliations}

V Glinkova, O Shevah, M Boaz, A Levine, H Shirin, Clinic of Hepatogastroenterology, "St Marina" University Hospital, Medical University, Varna, Bulgaria, and Epidemiology Unit, Pediatric Gastroenterology Unit and Department of Gastroenterology, The Edith Wolfson Medical Center, Sackler Faculty of Medicine, Tel-Aviv University, Israel

\section{REFERENCES}

1 Reddy KR, Kligerman S, Levi J, et al. Benign and solid tumors of the liver; relationship to sex, age, size of tumors, and outcome. Am Surg 2001;67:173-8.

2 Henson SW ir, Gray HK, Dockerty MB. Benign tumors of the liver. II. Hemangiomas. Surg Gynecol Obstet 1956;103:327-31.

3 Gandolfi L, Leo P, Solmi L, et al. A. Natural history of hepatic hemangiomas: clinical and ultrasound study. Gut 1991;32:677-80.

4 Porayko MK, Choudhary C. Benign neoplasms of the liver. Curr Treat Options Gastroenterol 2001:4:479-91.

5 Pietrabissa A, Giulianotti P, Campatelli A, et al. Management and follow-up of 78 giant hemangiomas of the liver. Br J Surgery 1996;83:915-18.
6 Saegusa T, Ito K, Oba N, et al. Enlargement of multiple cavernous hemangioma of the liver; association with pregnancy. Intern Med 1995;34:207-11.

7 Conter RL, Longmire WP jr. Recurrent hepatic hemangiomas. Possible association with estrogen therapy. Ann Surg 1988;207:115-19.

8 Morley JE, Myers JB, Sacks FS, et al. Enlargement of cavernous hemangioma associated with exogenous administration of estrogens. S Afr Med J 1974;48:695-7.

9 Torbenson $M$, Lee $\mathrm{JH}$, Choti $M$, et al. Hepatic adenomas: analysis of sex steroid receptor status and the Wnt signaling pathway. Mod Pathol 2002; 15:189-96.

10 Baker ER, Manders E, Whitney CW. Growth of cavernous hemangioma with puberty. Clin Pediatr 1985;24:596-8.

11 Trastek VF, van Heerden JA, Sheedy PF ii, et al. Cavernous hemangiomas of the liver: resect or observe? Am J Surg 1983;145:49-53.

12 Adami GL. Principles of pathology. Philadelphia: Lea \& Febiger, 1910.

13 Ochsner JL, Halpert B. Cavernous hemangioma of the liver. Surgery 1958;43:577-82.

14 Karhunen PJ. Benign hepatic tumors and tumor-like conditions in men. J Clin Pathol 1986;39:183-8.

15 Bruneton JN, Drauillard J, Fenart D, et al. Ultrasonography of hepatic cavernous hemangiomas. Br J Radiol 1983;56:791-5.

16 Grieco MB, Miscall BG. Giant hemangiomas of the liver. Surg Gynecol Obstet 1978;147:783-7.

17 Ishak KG, Rabin L. Benign tumors of the liver. Med Clin North Am 1975;59:995-1013.

18 Robbin SL. Pathologic basis of disease. Philadelphia: WB Saunders, 1974.

19 Takahashi T, Kuwao S, Katagiri H, et al. Multiple liver hemangiomas enlargement during long-term steroid therapy for myasthenia gravis. Dig Dis Sci 1998;43:1553-61.

20 Stauffer JQ, Lapinski MW, Honold DJ, et al. Focal nodular hyperplasia of the liver and intrahepatic hemorrhage in young females on oral contraceptives. Ann Intern Med 1985;83:301-6.

21 Vilgrain V, Uzan F, Brancatelli G, et al. Prevalence of hepatic hemangioma in patients with focal nodular hyperplasia: MR imaging analysis. Radiology 2003;229:75-9.

22 Wanless IR, Maudsley C, Adams R. On the pathogenesis of focal nodular hyperplasia of the liver. Hepatology 1985;5:1194-200.

23 Takagi A, Kajiura N, Tada Y, et al. Surgical treatment of non-specific inflammatory arterial aneurysms. J Cardiovasc Surg 1986;27:117-24.

24 Manthorpe R, Garbarsch C, Lorenzen I. Glucocorticoid effect on repair processes in vascular connective tissue. Morphological examination and biochemical studies on collagen RNA and DNA in rabbit aorta. Acta Endocrinol 1975;80:380-97.

25 Schnaper HW, McGowan KA, Kim-Schulze S, et al. Oestrogen and endothelial cell angiogenic activity. Clin Exp Pharmacol Physiol 1996;23:247-50

26 Jaggers DC, Collins WP, Milligan SR. Potent inhibitory effects of steroids in an in vitro model of angiogenesis. J Endocrinol 1996;150: 457-64.

\section{EDITOR'S QUIZ: GI SNAPSHOT}

\section{Answer}

From question on page 1344

The is a case of angiomyolipoma of the liver causing Budd-Chiari syndrome. Angiomyolipomas are benign tumors commonly seen in the kidneys. In this patient it was an incidental finding detected on ultrasound. However, the tumour mass impinged on the hepatic veins causing hepatic venous outflow obstruction. The diagnosis is suggested by the rather acute onset of abdominal pain and ascitis and the presence of caudate lobe hypertrophy on computerised tomography scan. The findings of both fat and vascular tissue on magnetic reonance imaging suggest angiolipoma. The image shown is hepatic venography which shows absence of flow in the hepatic veins. The patient had a guided biopsy which showed fat and vascular tissue with marked immunoreactivity to smooth muscle actin and HMB-45 antibody, which is highly suggestive of angiomyolipoma.

doi: 10.1136/gut.2003.31609 